\title{
Design and implementation of automatic test system based on network
}

\author{
Yong Xie, Zhen-Yu Wang, Si-Fang Liu and Li-Li Xia \\ China Satellite Maritime Tracking and Control Department, \\ Jiangyin 214431, China \\ Email: liumaster2006@163.com \\ *Corresponding author: Si-Fang Liu
}

\begin{abstract}
Based on the analysis of the interface relationship of each type of equipment, a set of automatic test system is developed by the unified interface. Four modules are designed for this purpose: I/O module, A/D module, protocol conversion module and microwave network module. The software of the host computer can control many different types of devices. The constructed system has reliable communication and operation/use convenient, good extension and for equipment to improve the level of information management, improve the ability of the task to carry out the test and made important contributions
\end{abstract}

Keywords: Airborne cooperative target; Automatic test; Function module; C\#.

\section{Introduction}

Due to the shipboard TT\&C equipment with the use of frequency band is wide, in order to meet the performance and testing of the accuracy calibration flight task needs in aircraft equipped with a large number of cooperative targets, including various frequency/mode response and beacon equipment. Is the ability to ensure that these carry out cooperative target test tasks, the daily necessary power and test work. Due to the different electrical characteristics of these cooperative target, the equipment volume has increased year by year, using test instruments and methods are not the same, if using the traditional manual test mode, not only time-consuming but also are not easy to the full state of the test.

To design a set of automatic test system, through the design of the corresponding function module, the cooperation goal is separated, and its interface is unified as the network interface, realize the automatic test of the host computer. 


\section{Overall System Design}

The object of the system design is to meet the working frequency band, working mode and beacon equipment of the ship equipment, as well as a variety of standard equipment for testing. For the standard instrument, including spectrum analyzer, signal generator and oscilloscope provides the GPIB interface, can adopt the GPIB [1] bus control; and response and these beacon cooperative target due to the different level of information, interface exist great differences, mainly in the form of the following kinds of interface: analog interface, serial interface and network interface. In order to facilitate the unified management of equipment, as well as to meet the requirements of the development of future equipment control, through the design of functional modules to achieve a unified device interface for the network interface, the composition of the system block diagram shown in figure 1.

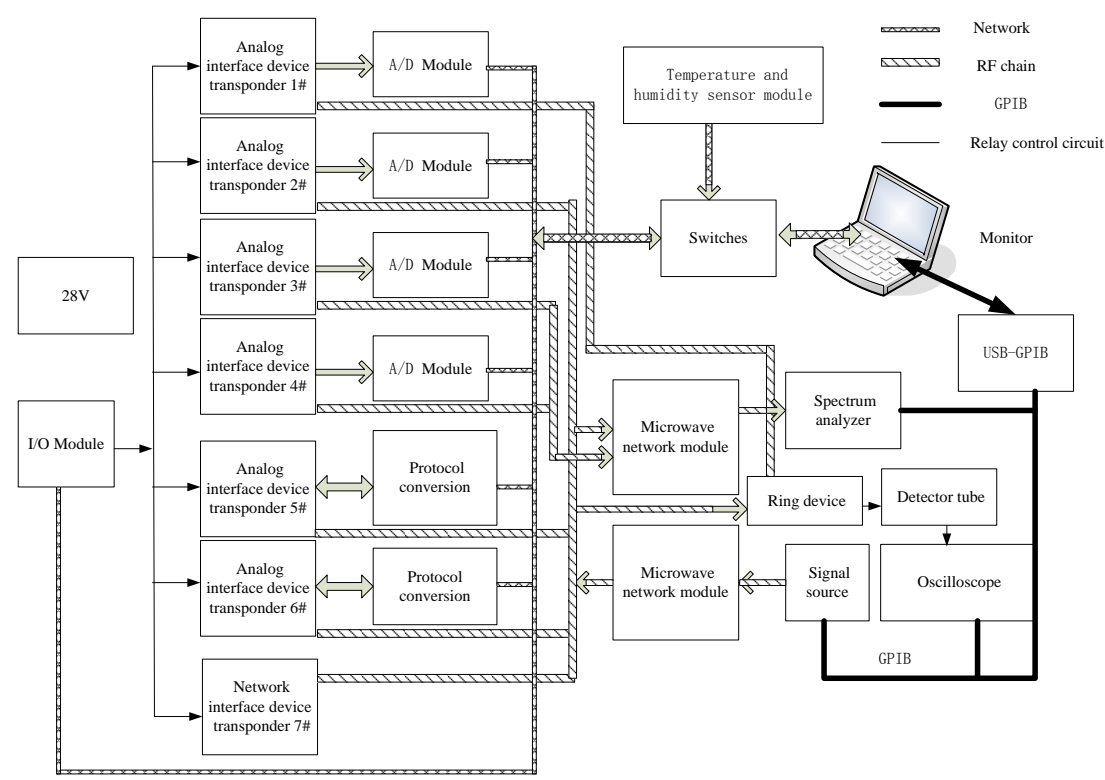

Fig. 1 Block diagram of system composition

The whole system consists of monitoring computer, standard instrument, various functional modules and cooperation targets, monitoring and controlling for scheduling and recording of the entire test process, just click the test button, can automatically complete the cooperative target automatic testing and data recording; standard instrument for transmitting, receiving and detecting signal to meet the electrical characteristics of cooperative target and it is controlled by GPIB bus; I/O module is used to realize the goal of cooperation on electric 
control, using the unified interface definition for equipment management; the $\mathrm{A} / \mathrm{D}$ module is used to complete the analog signal acquisition and transmission, using a device corresponding to a A/D module, A/D module and avoid equipment changes brought the inadaptability of microwave network [2]; the switching module is used for RF signal, avoid the RF link instrument brings less connection problems; circular polarizer and analyzer Tube wave to test the pulse signal, the complete RF signal loop back and pulse signal detection; protocol conversion module to complete and serial interface equipment protocol conversion, serial interface conversion as the interface of the network, also used a device corresponding to a conversion module; temperature and humidity sensor module is used for collecting environmental information, for the results of the tests provide data of temperature and humidity.

\section{Circuit Design of System Hardware}

In figure 1 , the system composition diagram shows, the hardware design of the system mainly includes four types of module design, namely the I/O module, A/D module, protocol conversion module and microwave network module, each module and monitoring computer interface for network interface, through monitoring computer to send / receive the provisions of the format of the frame, equipment electrical control, equipment parameter adjustment, equipment acquisition and RF link switching function is realized; at the same time, in order to ensure the integrity of the test elements design temperature and humidity sensor module, for acquisition of the test environment temperature and humidity. In order to reduce the difficulty of system hardware design, the design of the module network interface chip are using Neport module, through the Neport serial data is converted to UDP network data, so as to realize the module network.

\subsection{I/O module}

The main function of the I/O module to achieve the cooperation of the target plus /off control, the principle of the block diagram shown in figure 2. Power conditioning module to complete the DC $28 \mathrm{~V}$ power supply, through the LT1117-12 regulator for the 12V, and then through the LT1117-5.0 voltage regulator for $5 \mathrm{~V}$, the main control chip power supply. Due to the I / O control only needs a simple and not the number of external interface, so the main control chip adopts STC89C52, it through the serial port to receive Neport module data, changes in the level of corresponding I / O port control, realize the goal of cooperation of the power control by the driving chip to control the on-off of optocoupler relay (SRD-05VDC-SL-C). 


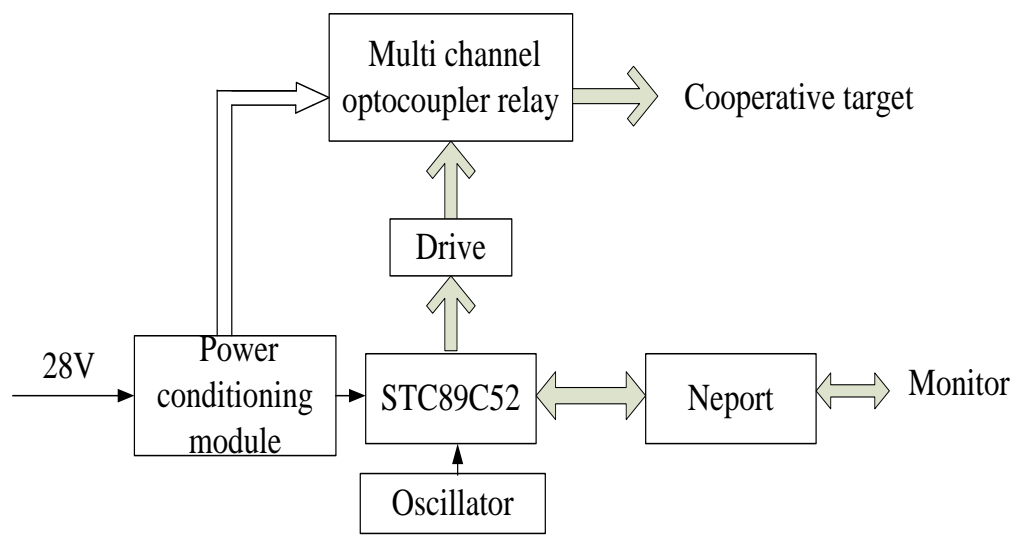

Fig. 2 Schematic diagram of I/O module

Control process for microcomputer monitoring select power equipment, to send UDP data to the I /O module, the data frame format: FF XX XX XX Fe, where FF as the frame header; Fe as the tail of the frame; XX data bits, in order to guarantee the communication reliability, STC89C52 port0, port1 and port2 use six port, to ensure the reliability of software processing. The Neport module converts the received UDP data packet to the serial port data, and then the STC89C52 is parsed to control the corresponding port.

\subsection{A/D module}



Fig. 3 Schematic diagram of A/D module 
Process control for cooperative target power, the analog signal through conditioning, acquisition give STC89C52, it the data packaged, the data frame format is: FF $\mathrm{X} 1 \mathrm{x} 2 \mathrm{xx} 1 \mathrm{XX} 2 \mathrm{xxx} 1 \mathrm{XXX} 2 \mathrm{Fe}$, the FF as the frame header; $\mathrm{Fe}$ as the tail of the frame; $\mathrm{x} 1, \mathrm{xx} 1$ and $\mathrm{xxx} 1$ as integer data, $\mathrm{X} 2, \mathrm{XX} 2, \mathrm{XX} 2$ small digital data, module Neport will the data conversion of UDP packets, sent to the monitoring computer.

\subsection{Protocol transform module}

Serial communication protocol to meet the requirements of other host computer control, so the serial control protocol is not the same, both common serial port protocol, there are Modbus protocol. In order to meet the functional requirements of the system design, we need to convert the serial protocol of the contract template to the network protocol, and design the protocol serial port module. The principle is shown in Figure 4. Due to meet the requirements of dual serial communication, where the main control chip using MSP430 [3], serial port 1 for the purpose of communication with the cooperation, serial port 2 for communication with Neport.

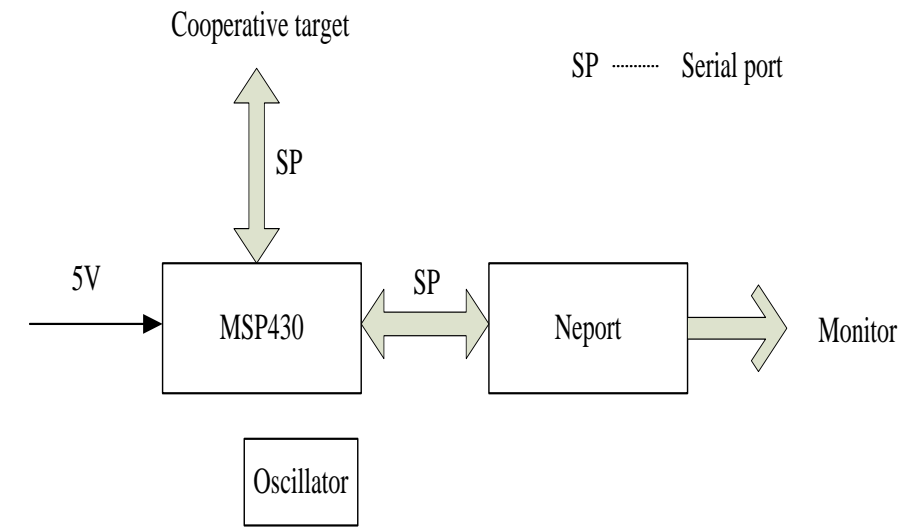

Fig. 4 Schematic diagram of protocol conversion module

According to the defined inside a cooperative target frame format and control protocol, MSP430 timer query data for process control, query results through serial port 2 send Neport conversion for UDP data and send it to computer monitoring; computer monitoring according to the control instruction sent UDP control packets, MSP430 after parsing and send them to the corresponding equipment. Its frame format is defined as: $\mathrm{XX} \mathrm{XX} \mathrm{DR...} \mathrm{CRC,}$ which Dr device address, each controlled cooperative targets were the only 
address, in order to distinguish between control; XX for data; CRC check data, due to the controlled more content, frame format is not exactly the same, but the basic frame format as above.

\subsection{Microwave network module}

Microwave network module main function is to achieve the radio frequency link switch, to meet the signal flow control of a small number of instruments, the principle of which is shown in figure 5 . The module uses $24 \mathrm{~V}$ power supply, is to meet the high frequency relay (QX6-618K002) power supply requirements, and its control for a simple I/O control. The power conditioning module is used to supply power for $24 \mathrm{~V}$ to STC $89 \mathrm{C} 52$ in $5 \mathrm{~V}$.

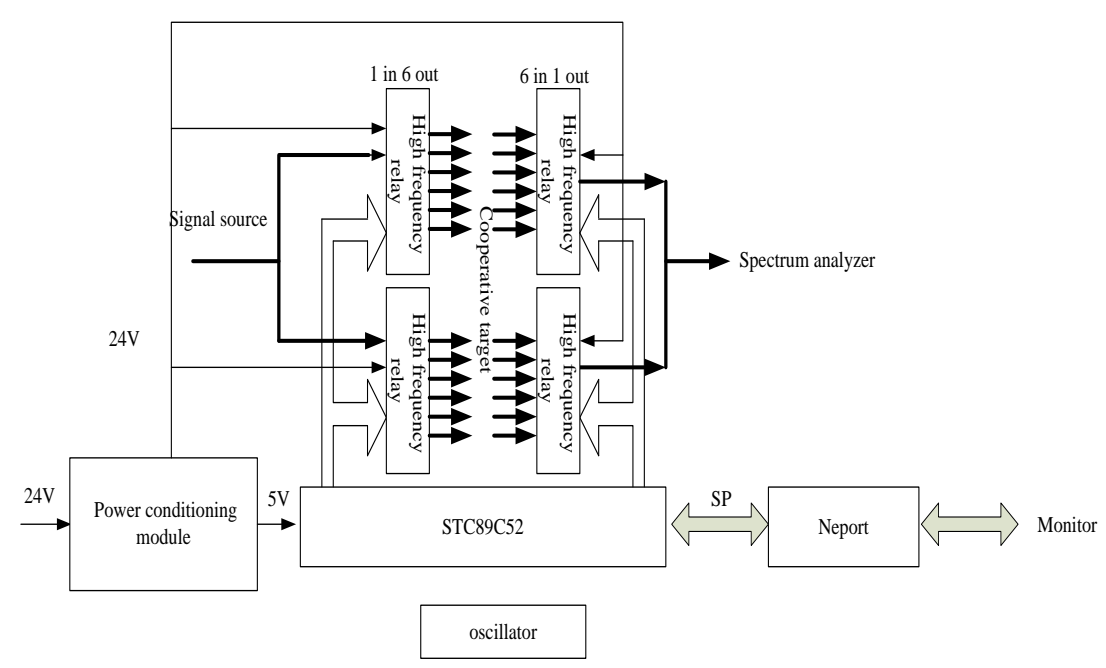

Fig. 5 Schematic diagram of microwave network module

Process control for microcomputer monitoring according to the testing content and object, sending a UDP packet, frame format is: FF XX XX XX XX $\mathrm{Fe}$, where FF as the frame header; $\mathrm{Fe}$ as the tail of the frame; $\mathrm{XX}$ for the data bits by four bytes is to avoid data bits in FF or Fe, and enhance software reliability.

\subsection{Temperature and humidity sensor module}

As a test project, its environmental condition is a factor that must be considered. Therefore, the design of the temperature and humidity sensor module is shown in Figure 6. Temperature and humidity sensors choose SHT75 chip, in the STC89C52 timing acquisition SHT75 temperature and humidity data, and the 
data package, sent to the Neport chip to achieve data networking. Its frame format is: $\mathrm{X} 1 \mathrm{X} 2 \mathrm{XX} 1 \mathrm{XX} 2 \mathrm{FE} \mathrm{FF}$, which FF for the frame header; FE for the end of the frame; $\mathrm{X} 1, \mathrm{XX} 1$ for the data of the entire digital, $\mathrm{X} 2, \mathrm{XX} 2$ for the data of the small digital [4].

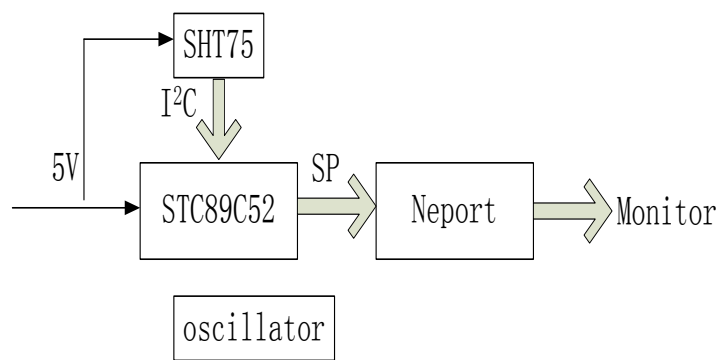

Fig. 6 Schematic diagram of temperature and humidity sensor module

\section{Software Design}

Function of computer monitoring and controlling software design mainly includes the depending on the test object are respectively arranged different working state parameters, and control the corresponding function module sets; acquisition function module of the state and parameter information, complete the display; general instrument GPIB control, setting up the parameters of the apparatus and acquisition spectrum analyzer and oscilloscope graphical data display and print.

Setting software for two work modes: full automatic testing and semi automatic test and in automatic test mode, monitoring software according to factor test to the test object is set and test, mainly for routine inspection, mainly transponder response models and beacon beacon mode signal quality inspection, etc.; in the semi automatic test pattern, the parameters for each of the objects were tuned and validated, mainly is the internal parameters of the various test objects, such as the spread spectrum code group settings and test.

The key technologies of software development include: XML file reading and writing, Socket network communication, database technology [5] and so on. The XML file is used to complete the configuration information and adjust the settings, including the state of the instrument parameters, cooperative target normal state parameters is initiated by the system test source information; socket network communication, from the XML file to read data frame structure, for the completion of the UDP packets sent and analytical; database technology is used to store the testing results, easy afterwards analysis and statistics, and a graphic display/print, word processing documents, and so on. 


\section{Conclusions}

The system design and implementation of a large number of various types of airborne cooperative target automatic test, to meet the needs of the daily maintenance of equipment, improve the level of information technology equipment management. Through the hardware design of the system, we can know that the system has a very high cost performance, good real-time performance, easy control, good expansibility and so on. The design method of the "separated object unified interface", which is used in the system design, has a high reference value for the development of other similar control fields.

\section{References}

[1]. TI.CC2530 Data manual [EB].Texas Instruments, Inc. San Diego, California USA, 2007.

[2]. Wang Yonghua. Field Bus Technology and Application [M]. Beijing: Machinery Industry Press, 2008. (In Chinese)

[3]. Ma Zhiqiang. Range of Geodesy [M].Beijing: National Defence Industry Press, 2004. (In Chinese)

[4]. TI.CC2591 Data manual [EB].Texas Instruments, Inc. San Diego, California USA, 2007.

[5]. Huang yuli. Design of WSN Node Communication Module Based on CC2531+CC2591 [J]. Application of single chip microcomputer and embedded system, 2011, 1:71-73. (In Chinese) 